CERN EP/2000-152

December 5, 2000

\title{
Cosmic multi-muon events observed in the underground CERN-LEP tunnel with the ALEPH experiment
}

\author{
V. Avati, L. Dick ${ }^{\mathrm{a})}$, K. Eggert, J. Ström ${ }^{\mathrm{b})}$, H. Wachsmuth ${ }^{\mathrm{c})}$ \\ European Laboratory for Particle Physics (CERN), Geneva, Switzerland \\ S. Schmeling, T. Ziegler \\ Institut für Physik, Johannes Gutenberg-Universität Mainz, Germany \\ A. Brühl, C. Grupen \\ University of Siegen, Siegen, Germany
}

\begin{abstract}
Multimuon events have been recorded with the ALEPH-detector, located $140 \mathrm{~m}$ underground, in parallel with $\mathrm{e}^{+} \mathrm{e}^{-}$data taking. Benefitting from the high spatial and momentum resolution of the ALEPH tracking chambers narrowly spaced muons in high multiplicity bundles could be analysed. The bulk of the data can be successfully described by standard production phenomena. The multiplicity distribution favors, though not with very high significance, a chemical composition which changes from light to heavier elements with increasing energy around the "knee". The five highest multiplicity events, with up to 150 muons within an area of $\sim 8 \mathrm{~m}^{2}$, occur with a frequency which is almost an order of magnitude above the simulation. To establish a possible effect, more of these events should be recorded with a larger area detector.
\end{abstract}

(Submitted to Astroparticle Physics)

\footnotetext{
a) Retired from CERN

b) visitor from ARCADA Polytechnic, Helsinki, Finland

c) now at Mainz University
} 
The distributions of muons accompanying extensive air showers, initiated by primary cosmic rays interacting in the upper atmosphere, have been studied with large-area arrays close to the surface of the ground [1-3] and deep underground [4-7]. A primary goal of these experiments has been to better understand the mass composition and energy spectrum of primary cosmic ray nuclei, particularly in the energy range of 1 to $100 \mathrm{PeV}$. The data presented here are for muons observed at an intermediate depth, corresponding to a vertical muon threshold energy of $70 \mathrm{GeV}$. The primary composition is known from direct measurements with satellite and balloon-borne experiments up to about $10^{14} \mathrm{eV}$ due to the rapidly falling flux vs. energy; higher energies require larger detector areas and longer exposure times than have been possible with instrumentation flown at the top of the atmosphere. Better knowledge of composition vs. energy may aid in interpreting the origin and acceleration mechanism of cosmic rays in this energy range.

The cosmic ray community has been studying air showers for decades, seeking to learn the primary composition at energies above those accessible to direct observation. The problem is that the determination of the primary composition is inextricably linked to the dynamics of the first interactions, as far as air shower observables are concerned. The Monte Carlo models which simulate the primary interaction are based upon experimental observations at energies many decades below those discussed here and additionally have to use elementary-particle models to describe the interactions at the energies of interest. There are several Monte Carlo models which have been used to simulate this interaction - VENUS, QGSJET, SIBYLL, etc [8]. Different experiments using the above Monte Carlo simulations have reported very different mass compositions in the "knee" region [9]. For example, the deduced composition appears significantly different for ground hadron measurements and for electron-muon measurements [10].

Ultimately, one must 'tune' the Monte Carlo to agree with all reliable data; the greater the diversity of measurements included, the better the test; e.g. hadron measurements, muon distributions, electromagnetic characteristics, and the combinations of these different variables. New high energy effects in the primary interactions not included in any current Monte Carlos, may also reflect themselves in the observations. As examples, coherent effects in nucleus-nucleus interaction, coherent pion production, disordered chiral condensate (DCC) states [11], anomalous heavy flavor production in the very forward region [12], etc. could prove to be important. It is in this spirit that the muon data in the intermediate energy range reported here are being studied.

The use of the sophisticated and large underground detectors at CERN for cosmic ray studies has been previously discussed by several groups, e.g. UA1 [13] and LEP detectors [14, 15]. They offer, for the first time, the opportunity to provide large sensitive areas with magnetic analysis which allow a precise determination of the directions of the cosmic ray muons as well as their momenta up to the order of several hundred $\mathrm{GeV}$, even for large muon multiplicities. The underground locations of the LEP detectors, between 30 and $140 \mathrm{~m}$, are ideal for muon measurements: the electromagnetic and hadronic components of the air showers of cosmic rays are fully absorbed by the ground, and the muon momentum cut-off is between 15 and $70 \mathrm{GeV}$ for vertical incidence, depending on the detector depth. A muon momentum of about $70 \mathrm{GeV}$ is well-matched to the energy at which charged pions have similar probabilities for interaction and decay at the top of the atmosphere. For example, the decay mean free path of a $100 \mathrm{GeV}$ pion is $5.5 \mathrm{~km}$ which is equal to the interaction mean free path of a pion in air produced in the upper atmosphere at a pressure of about $100 \mathrm{~g} / \mathrm{cm}^{2}$.

Consequently, these measurements may be more sensitive to very different convolutions 
of particle production than those performed by other experiments. Hence cosmic ray experiments using the infrastructure of the LEP tunnel are clearly complementary to previous and existing cosmic ray experiments, most of which are either much deeper underground or located on or very near the surface. For the deep underground experiments, such as MACRO [4], FREJUS [5,6] and SOUDAN [7] the momentum cut-off is of order of one TeV, whereas for surface experiments, such as KASCADE [1] and CASA-MIA [2] it is of the order of 2-4 GeV.

High-energy muon showers might also be used as a telescope to look for point sources in our galaxy and beyond since the directional accuracy is excellent, typically 3 mrad for a bundle of 20 muons. In fact high energy muons retain with good accuracy the arrival direction of the parent particles and thus direction of the primaries. The arrival directions of the charged primaries in our energy range (less than $10^{17} \mathrm{eV}$ ) are strongly distorted by galactic magnetic field, but high energy neutrinos, neutrons, gamma and protons from nearby sources give the direction to the source.

\section{The ALEPH experimental set-up}

The contributions of this experiment to the study of multi-muon bundles from cosmic rays result from the superb characteristics of the ALEPH detector [16] which is located at the deepest LEP point, $140 \mathrm{~m}$ underground, corresponding to a momentum cut-off of $70 \mathrm{GeV}$ for vertical muon incidence. The large Time-Projection-Chamber (TPC) inside a solenoidal field of 1.5 Tesla provides excellent pattern recognition, tracking resolution and momentum determination, and is a unique tool giving information previously unavailable in cosmic ray studies. For example, muon bundles with densities of up to 20 muons $/ \mathrm{m}^{2}$ could easily be resolved and $>30 \%$ of the muons could be momentum analyzed with a precision $\Delta p / p^{2}$ of better than $0.1 \% / \mathrm{GeV}$. The tracking resolution of the TPC enables the identification of muon induced showers (electromagnetic and hadronic) which otherwise could simulate a high-density core of muons. The acceptance of the solenoidal TPC is almost isotropic, as can be deduced from Fig. 1 where the effective TPC area is plotted versus the azimuthal angle and for different zenith angles. The TPC is surrounded by electromagnetic and hadronic calorimeters with tracking capabilities and by an outer shell of muon chambers.

As an example and to illustrate the ALEPH detector capabilities, one of the highest multiplicity muon events is displayed in Fig. 2 with views along and perpendicular to the LEP ring and also perpendicular to the muon shower direction. The excellent resolution of the TPC easily resolves close-by muon tracks and enables an angular determination to better than $5 \mathrm{mrad}$, the average multiple scattering angle of the muons in the $140 \mathrm{~m}$ overburden. Further information about the muon density is given by the forward calorimeters and muon chambers. The 76 recorded tracks in the TPC appear as points in the plane perpendicular to the muon bundle axis for those muons which are parallel to the shower axis. As seen in Fig. 2, the muons are almost parallel and are distributed isotropically over an area of $4 \times 3 \mathrm{~m}^{2}$. The large muon density of about 6 muons $/ \mathrm{m}^{2}$ extends further into the forward calorimeters.

\section{Data selection and reconstruction}

Standard LEP triggers of ALEPH are also sensitive to cosmic ray muons, particularly multi-muon events, if they occur within a gate of a few microseconds centered on the electronpositron beam-crossing times. The cosmic events were triggered by the energy deposition of the muons in the electromagnetic and hadronic calorimeters. They were selected from the LEP triggers by identifying events without a reconstructed vertex, with at least one well-defined track with momentum above $5 \mathrm{GeV} / \mathrm{c}$ and no track from the $\mathrm{e}^{+}-\mathrm{e}^{-}$vertex region. This last vertex requirement was not applied to high multiplicity muon events in order to avoid losing 


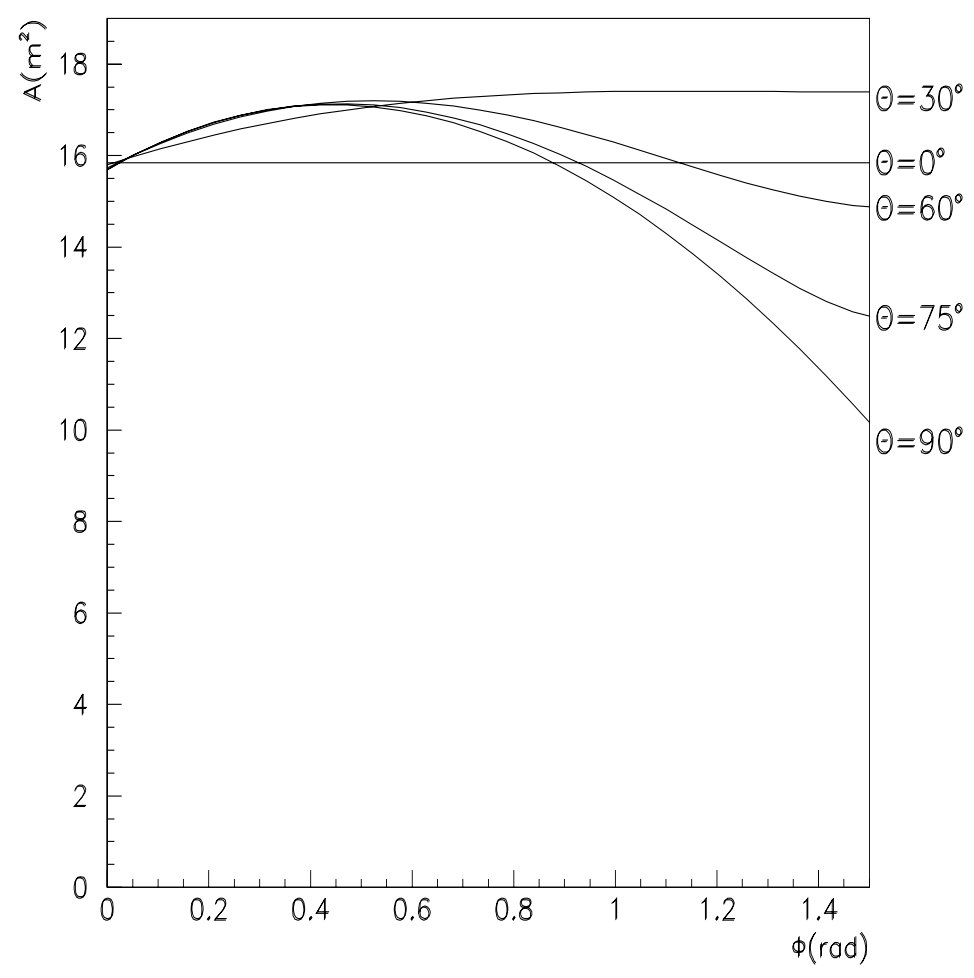

Figure 1: Dependence of the effective TPC area as function of the azimuthal angle for different zenith angles, including the effects of the track selection criteria.

such events. All muons in the event, with a track length larger than $0.5 \mathrm{~m}$ and at least 4 hits per track, were reconstructed and their common arrival time was determined either from the electromagnetic calorimeter or from tracks crossing the central membrane of the TPC. The flat time distribution of the events over a time interval of $2.5 \mu \mathrm{sec}$ around the beam-crossing every $22.3 \mu \mathrm{sec}$ determined the average duty cycle of $11.5 \pm 1 \%$ for cosmic ray data taking. The tracking chambers in the calorimeter and the muon chambers have a much shorter sensitive time $(<1 \mu \mathrm{sec})$ and therefore information from these devices is often not recorded.

About 580000 cosmic ray events were selected from the LEP data taking periods during the years 1997 to 99 , corresponding to an effective data taking time of $1.7 \cdot 10^{6} \mathrm{sec}$. Tab. 1 summarizes the statistics of the years 1997 - 99, i.e. the data taking time, the duty cycle and the number of events after all cuts. After a consistency check of the individual data sets, the data from the different years were combined.

A representative sub-sample of the data was scanned, as were all events with multiplicity greater than 6 , in order to remove beam-gas and muon interactions that can simulate highmultiplicity events. An event with a muon interaction in the ALEPH detector, depositing more than $50 \mathrm{GeV}$ in the hadronic calorimeter and producing many tracks in the TPC, is shown in Fig. 3. It demonstrates that good tracking is absolutely mandatory to distinguish muon induced showers from muon bundles.

The muon momentum is determined by a track fit. Due to the optimization of the ALEPH geometry for LEP events, the best accuracy is obtained for tracks passing close to the vertex region. For about $30 \%$ of the reconstructed tracks the maximum detectable momentum exceeded $1 \mathrm{TeV}$. These tracks were used for the muon momentum distributions. 

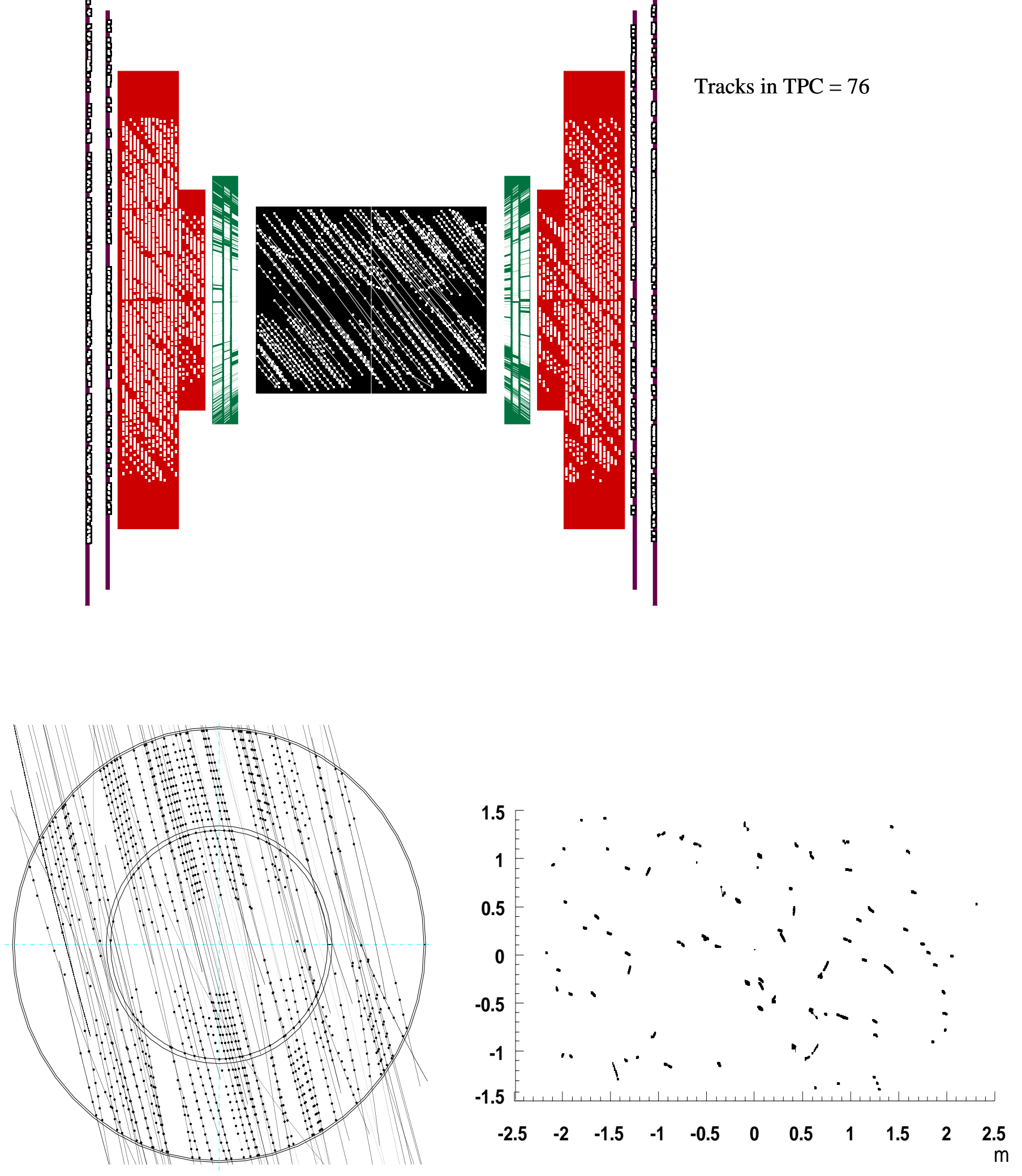

Figure 2: A high multiplicity event (76 tracks) in three different views: perpendicular and along the LEP ring and along the muon shower direction. 


\begin{tabular}{|c|c|c|c|c|}
\hline & 97 data & 98 data & 99 data & 97+98+99 data \\
\hline \hline Run time $\left(10^{6} \mathrm{sec}\right)$ & 3.49 & 5.85 & 5.68 & 15.02 \\
\hline Effective run time $\left(10^{6} \mathrm{sec}\right)$ & .3834 & .6816 & .6817 & 1.7467 \\
\hline Number of events $\left(10^{3}\right)$ & 151 & 222 & 211 & 584 \\
\hline
\end{tabular}

Table 1:

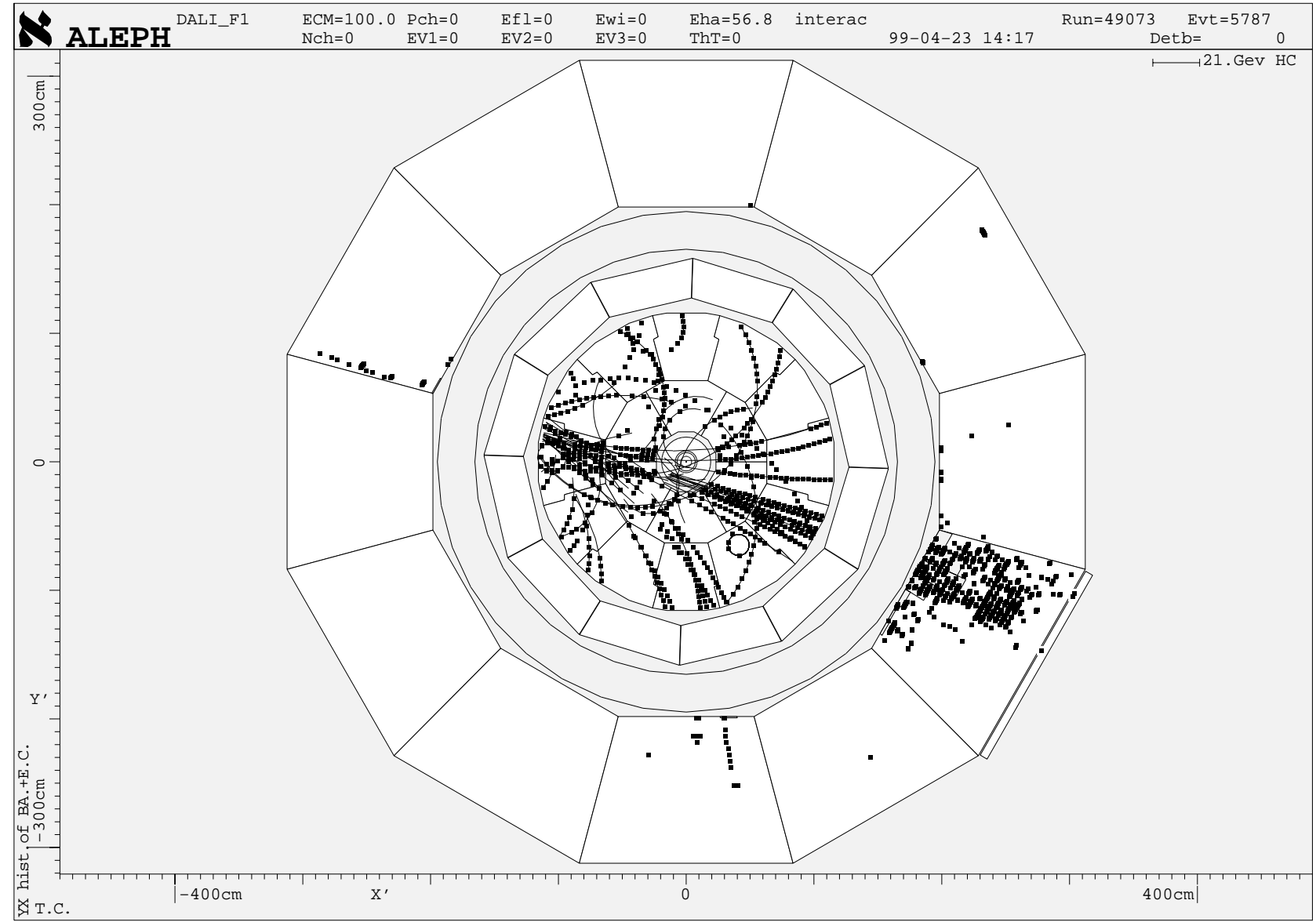

Figure 3: A muon interaction in the ALEPH detector, producing a cascade with energy deposition in the electromagnetic and hadronic calorimeters. 
For quantitative analysis, the ALEPH data were compared with the expectations from cosmic ray air shower simulations. The current state of the art is represented by CORSIKA [8], a sophisticated simulation program developed in recent years by the KASCADE group in Karlsruhe. The interactions of primary cosmic ray nuclei (proton through iron) may be simulated by one of several Monte-Carlo programs: VENUS, SIBYLL, QGSJET, Dual Parton,...[17]. These primary interaction models use as an input the data from numerous accelerator experiments which are then extrapolated to higher energies with some theoretical prejudice. For the comparison with our data we have used the QGSJET model.

The resulting air showers initiated by the reaction products of the first interaction are simulated by the Corsika Monte-Carlo, following the evolution of the hadron, muon and electromagnetic distributions to the observation level. The Corsika program is dominated by lowenergy interactions, within the domain well known from existing accelerator data. For this analysis, only the hadronic component with hadrons decaying into muons was followed since the muon contribution from the electromagnetic component is negligible.

A momentum cut of $70 \mathrm{GeV} / \cos \theta$, where $\theta$ is the zenith angle of the muon, was applied to account for the energy loss in the overburden of ALEPH. This momentum cut resulted from a GEANT calculation which took into account the detailed composition of the overburden and the geometry of the experimental hall, including the access shafts. The correction due to the access shafts on multi-muon events was negligible. Monte Carlo samples with statistics comparable to that of the data were simulated for two incident particle types: protons and Fe-nuclei. To obtain good statistics over the entire relevant energy range $\left(10^{12}-10^{18} \mathrm{eV}\right)$, the interactions were generated in primary energy bins and weighted according to the cosmic ray spectrum. The shower axes were scattered over an area of $200 \times 200 \mathrm{~m}^{2}$ centered around the ALEPH detector and the muons hitting the sensitive area of the TPC were registered.

\section{$5 \quad$ Results}

In principle, the transverse size of muon bundles and the muon multiplicity distributions are sensitive to the mass number $A$ of the initiating primaries. Fig. 4 shows the radial muon distribution $\left(\mathrm{p}_{\mu}>70 \mathrm{GeV} / \cos \theta\right)$ produced by proton and iron induced air showers for various primary energies, as predicted by the QGSJET CORSIKA simulation. The basic features of these distributions are:

- the central muon density grows almost linearly with the primary energy and depends only slightly on the mass of the primary particle for energies above $10^{15} \mathrm{eV}$

- the muon density falls rapidly with radius from the shower centre, dropping by an order of magnitude over 15 - $20 \mathrm{~m}$. Its radial dependence is almost independent of energy for $\mathrm{E} \geq 10^{15} \mathrm{eV}$

- for primary energies exceeding $3 \cdot 10^{14} \mathrm{eV}$ the radial dependence of the muon density becomes flatter with increasing mass of the primary particle, leading to twice as many muons for iron induced showers compared to proton-induced events of the same total energy.

Whereas the TPC is too small to contain a sizeable fraction of the muon bundle and hence to measure the radial dependence of the muon density it is the ideal detector to measure the local muon density at the highest observed values of 20 muons $/ \mathrm{m}^{2}$ and above. Fig. 5 shows the measured distribution of the muon multiplicity in the TPC and the QGSJET CORSIKA simulation for proton and iron primary particles, absolutely normalized to the effective running time. Up to a multiplicity of 20, the proton curve describes the observed data well over several orders of magnitude, indicating that the primary spectrum is dominated by light elements at 


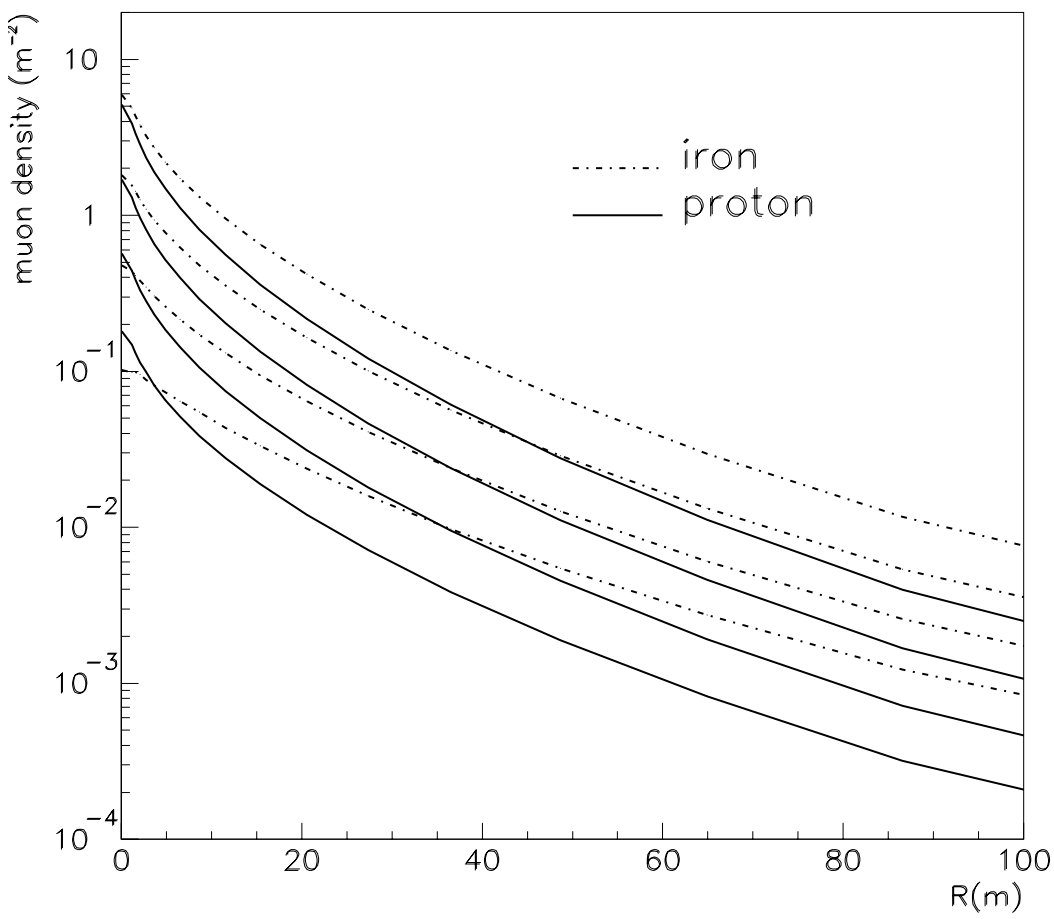

Figure 4: CORSIKA Monte-Carlo simulations of the muon density $\left(\mathrm{p}_{\mu}>70 \mathrm{GeV}\right)$ for proton and iron induced vertical showers of various energies as a function of the radial distance from the shower center. The curves from the bottom to the top are for primary energies of $3 \cdot 10^{14}$, $10^{15}, 3 \cdot 10^{15}, 10^{16} \mathrm{eV}$.

energies corresponding to these multiplicities. The discrepancy in the single muon rate is due to the low efficiency of the LEP triggers for single muons; this efficiency approaches $100 \%$ only if there are more than two muons in the TPC. At larger multiplicities there is evidence for a transition to the iron curve. Iron induced showers are more effective in producing muons (see Fig. 4) since they interact higher in the atmosphere and also produce larger pion multiplicities. While the simulation agrees with the data over a wide multiplicity range, it fails to describe the highest multiplicities, even under the assumption of a pure iron composition. This is better demonstrated with the integral distribution in Fig. 6, which is plotted on a log-log scale for shower zenith angles below and above $30^{\circ}$. It is worth noting that four of the five highest multiplicity events have zenith angles beyond $30^{\circ}$. The highest multiplicity event, displayed in Fig. 7, contains about 150 muons in only one half of the TPC since the other half could not be read-out due to data overflow. Extrapolated to the full acceptance of the TPC about 250-300 muons would be expected over the area of $16 \mathrm{~m}^{2}$, i.e. $15-20$ muons $/ \mathrm{m}^{2}$. Tab. 2 summarizes the characteristics of the five highest multiplicity events. An estimate of the primary energy was made under the assumption that the shower center is in the TPC. The energy was calculated assuming proton-induced showers and would be $40 \%$ lower for iron. If the shower cores were further away, the energies would be even larger.

The muon density grows almost linearly with the primary energy and hence multiplicity bins are equivalent to energy bins. The contribution of different energy intervals of the primary spectrum to the muon multiplicity in the TPC is shown in Fig. 8 for proton induced showers. Multiplicities between 5 and 20 are sensitive to the energy spectrum around $10^{15}$ and $10^{16} \mathrm{eV}$, 


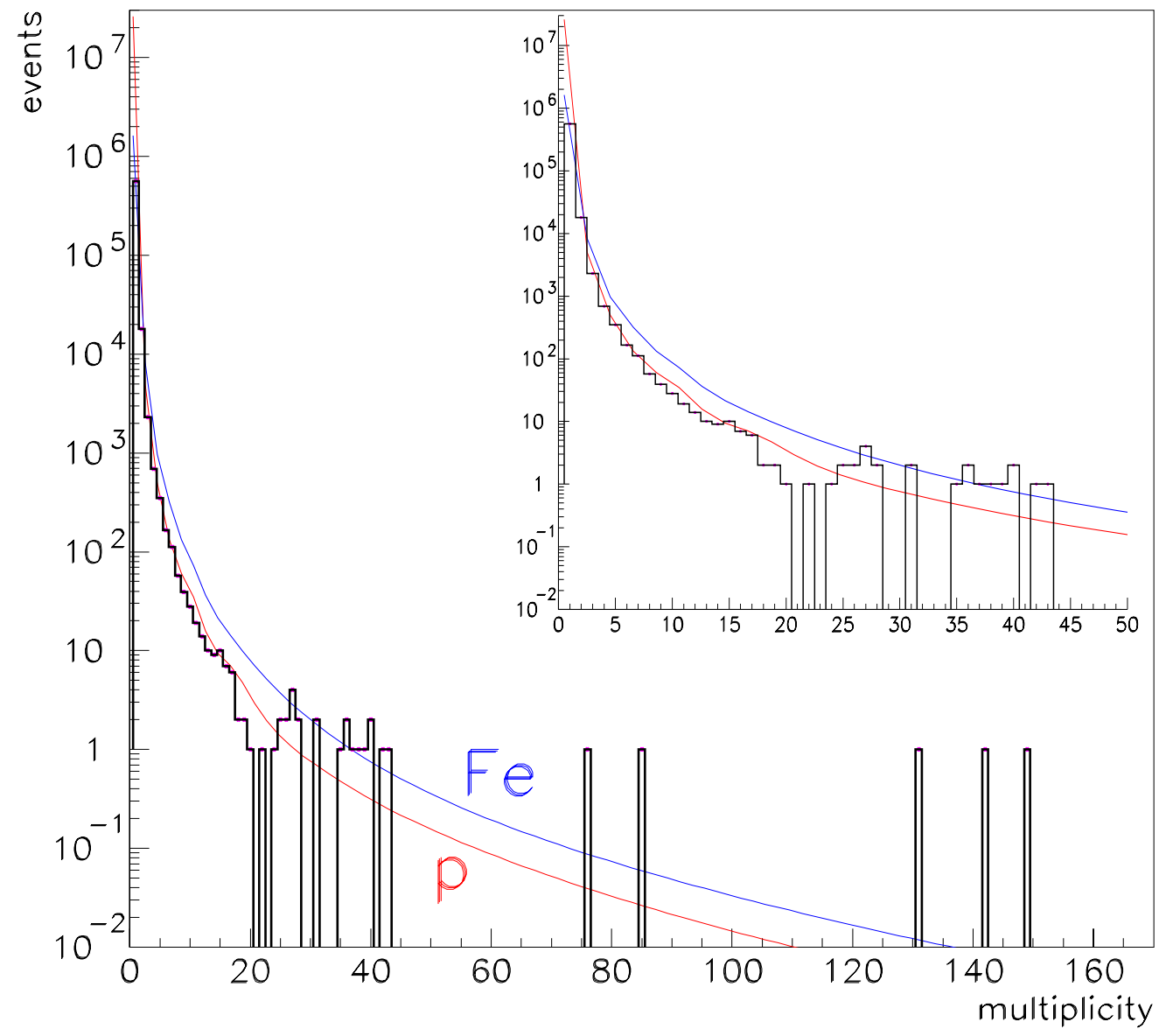

Figure 5: Multiplicity distribution of muons in the TPC compared to CORSIKA simulations for $p$ and $\mathrm{Fe}$ as primary particle.
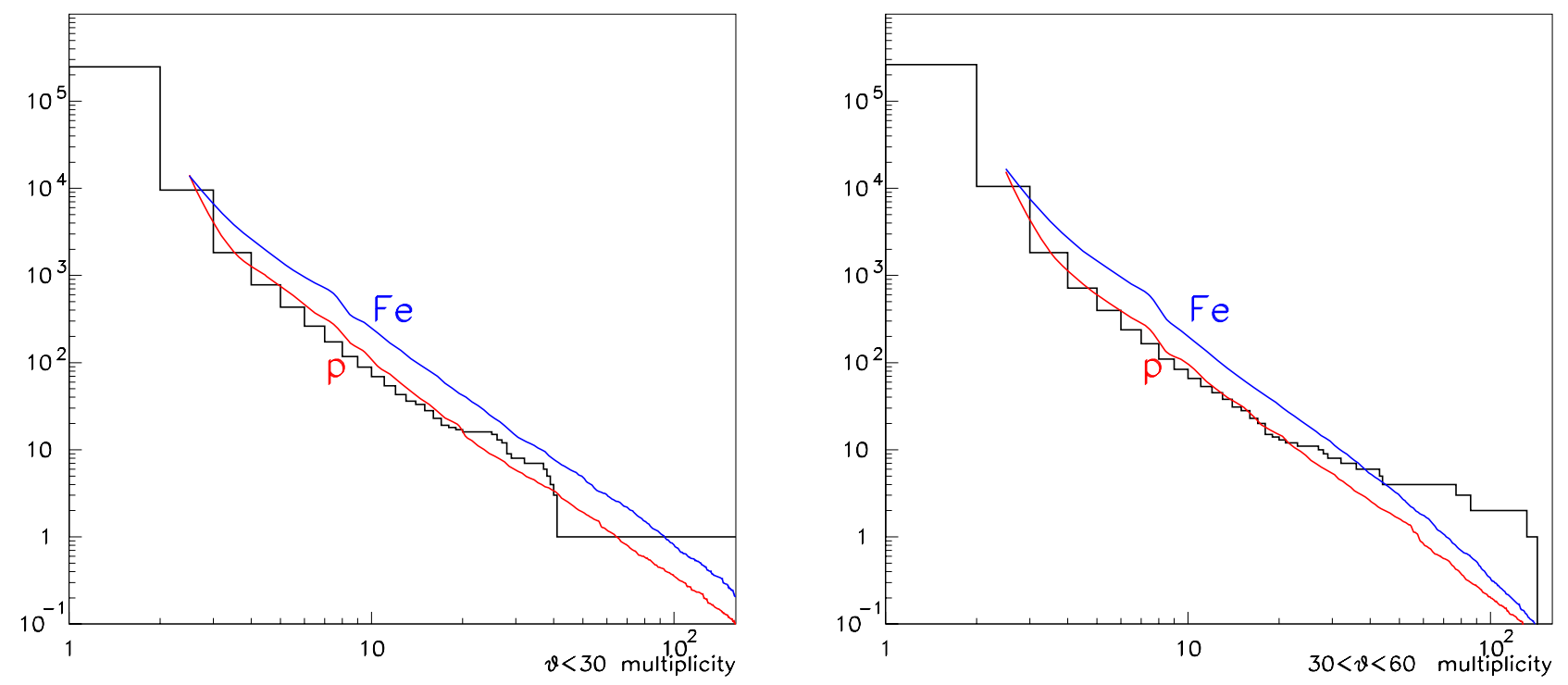

Figure 6: Integral multiplicity distributions of muons in the TPC for two different zenith angle intervals $\left(\theta<30^{\circ}\right.$ and $\left.30^{\circ}<\theta<60^{\circ}\right)$ compared to CORSIKA simulations for $p$ and Fe. Power law fits of the M.C. curves yield an exponent of about -1.8 . 

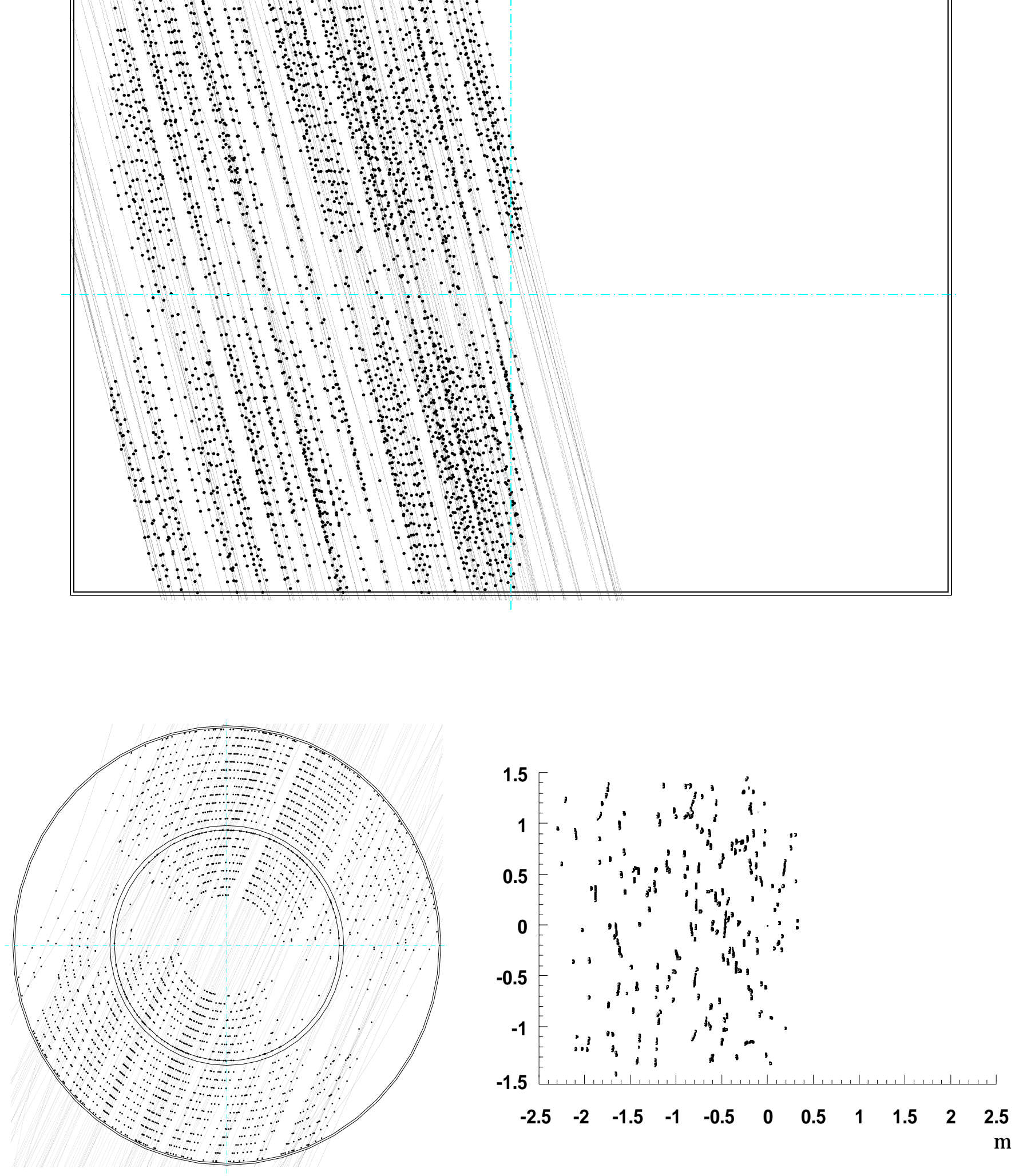

Figure 7: The event with the highest multiplicity (about 150 tracks, in half of the TPC) in three different views: longitudinal, transverse and along the shower axis. Reconstructed tracks are shown as lines 


\begin{tabular}{|c|c|c|c|}
\hline event & $\begin{array}{c}\text { muon } \\
\text { density }\left(\mathrm{m}^{-2}\right)\end{array}$ & $\begin{array}{c}\text { zenith } \\
\text { angle }\left(^{\circ}\right)\end{array}$ & $\begin{array}{c}\text { primary } \\
\text { energy }(\mathrm{eV})\end{array}$ \\
\hline $97-\mathrm{a}$ & 4.75 & 40.8 & $3 \cdot 10^{16}$ \\
$97-\mathrm{b}$ & 5.3 & 37.7 & $3 \cdot 10^{16}$ \\
$97-\mathrm{c}$ & 8.9 & 40 & $6 \cdot 10^{16}$ \\
$98-\mathrm{a}$ & 8.2 & 48.6 & $7 \cdot 10^{16}$ \\
$98-\mathrm{b}$ & 18.6 & 27 & $10^{17}$ \\
\hline
\end{tabular}

Table 2: Characteristics of the highest multiplicity events. The primary energy was estimated by assuming the shower center to be close to the TPC and taking into account the zenith angle.

thus covering the interesting region around and above the knee. Multiplicities above 30 arise predominantly from primary energies above $10^{16} \mathrm{eV}$. Measurements of the particle composition as well as theoretical prejudices about the acceleration mechanism of cosmic rays suggest an increase of the heavy component above $10^{16} \mathrm{eV}$, not in contradiction with our multiplicity spectrum. Power-law fits to the integral multiplicity distribution in Fig. 6 yield an exponent of -1.8 which is close to the one for the primary energy spectrum, consistent with the correlation between muon multiplicity and primary energy.

With the use of the LEP detectors, it is possible for the first time to measure the momenta in cosmic ray multi-muon events over a sizeable detector area. The ALEPH detector is optimized for tracks coming from the vertex and hence only muons passing close to the vertex region are well measured. We applied a momentum resolution cut of $\Delta p / p^{2}<10^{-3} / \mathrm{GeV}$ which is fulfilled by $35 \%$ of the tracks. The efficiency of this cut is independent of the obtained multiplicity. Scaled with the above efficiency factor, the momentum distribution up to $1 \mathrm{TeV}$ is given in Fig. 9 for different bins in multiplicity. The momentum distribution is flatter for multi-muons than for single muons. This is well described by the simulation which is absolutely normalized. The higher momenta in multi-muon events can be qualitatively understood since these muons are closer to the shower core. They also arise from higher primary energies and originate from an earlier stage of the shower development.

The primary particle direction is determined by an average over all muon directions, which are parallel to within a few degrees within a single event. As an example, the distribution of the muon angles with respect to the bundle axis is plotted in Fig. 10 for the five highest multiplicity events. The angular spread is typically $10 \mathrm{mrad}$ in agreement with the expected multiple scattering. The zenith angle distribution of the showers follows a power law in the variable $\cos \theta$, where the exponent is close to 2 for single muons. For multi-muon events with more than two muons this exponent is 3.2 as can be deduced from Fig. 11, where the measured $\cos \theta$ distribution is compared to the CORSIKA simulation. It should be recalled that the muon momentum cut-off increases as $1 / \cos \theta$ due to the larger overburden.

In order to search for point-like sources in the universe, the muon bundle directions have been transformed into galactic coordinates. The events with multiplicity $\mathrm{N} \geq 3$ and the high multiplicity ones $(\mathrm{N} \geq 20)$ are plotted in Fig. 12. The events do not cluster or point to known photon sources in our galaxy or nearby galaxies $(<100 \mathrm{Mpc})$. Some of the known galactic and extragalactic sources are marked with letters. 


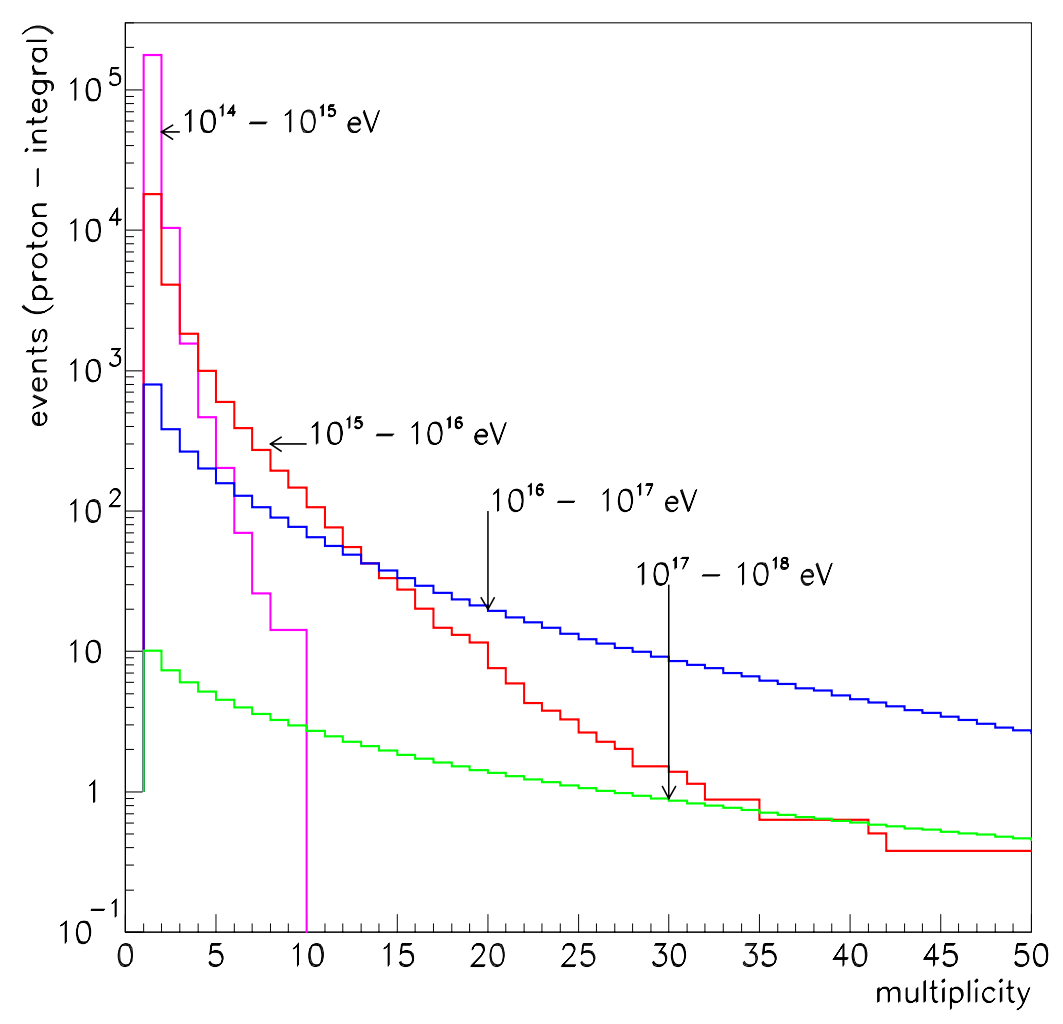

Figure 8: Contribution of the different energy intervals of the primary spectrum to the muon multiplicity for proton initiated showers.

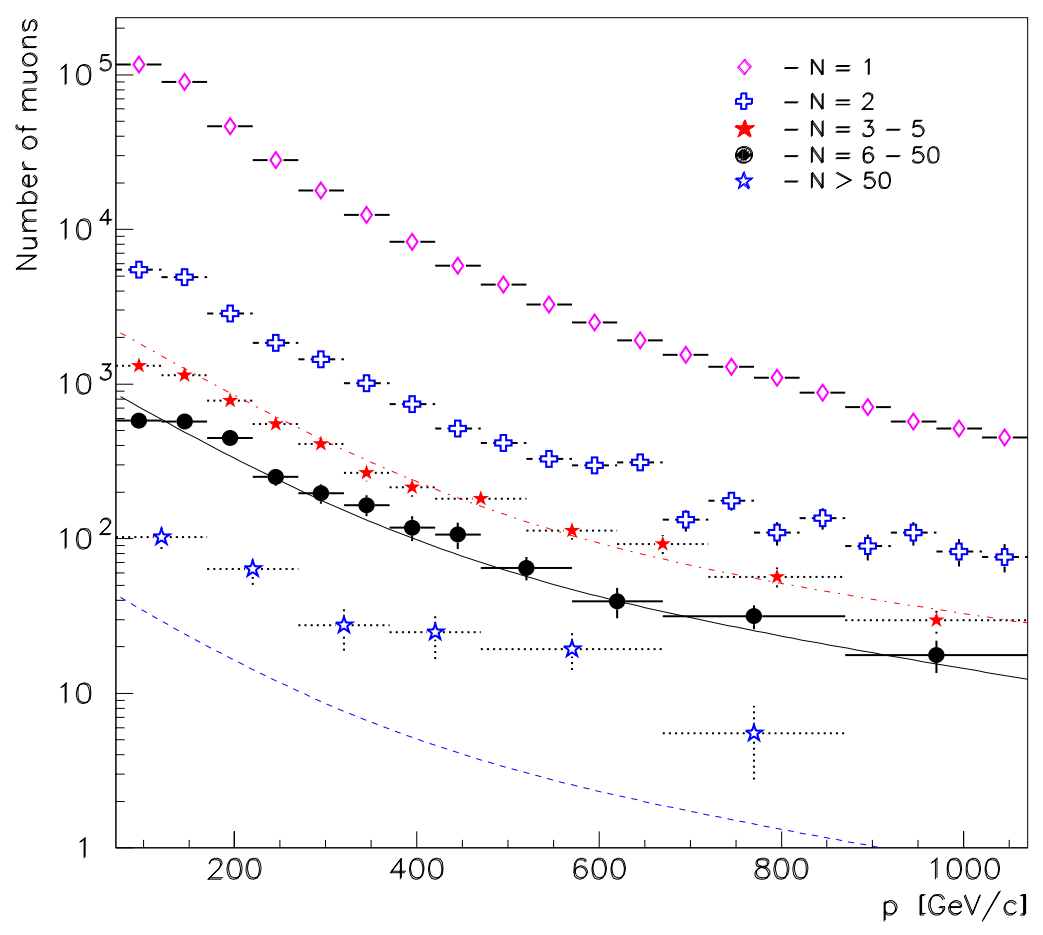

Figure 9: Momentum distributions of muons in the ALEPH TPC for several multiplicity bins. The energy loss in the overburden has been compensated for by adding $70 \mathrm{GeV} / \cos (\theta)$ to the measured momenta. The three lines for the multiplicity bins $N=3-5,6-50,>50$ represent the simulation for proton primaries, absolutely normalized 


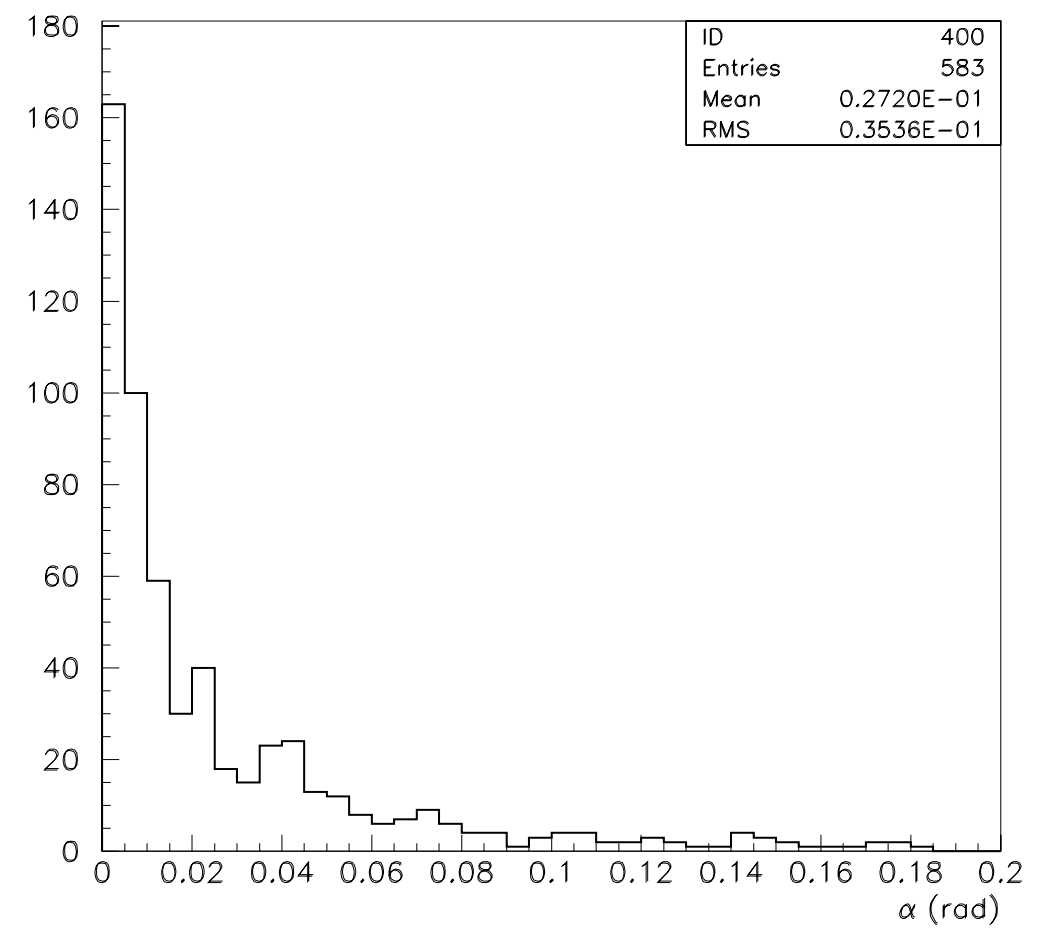

Figure 10: Distribution of muons angles with respect to the bundle axis.

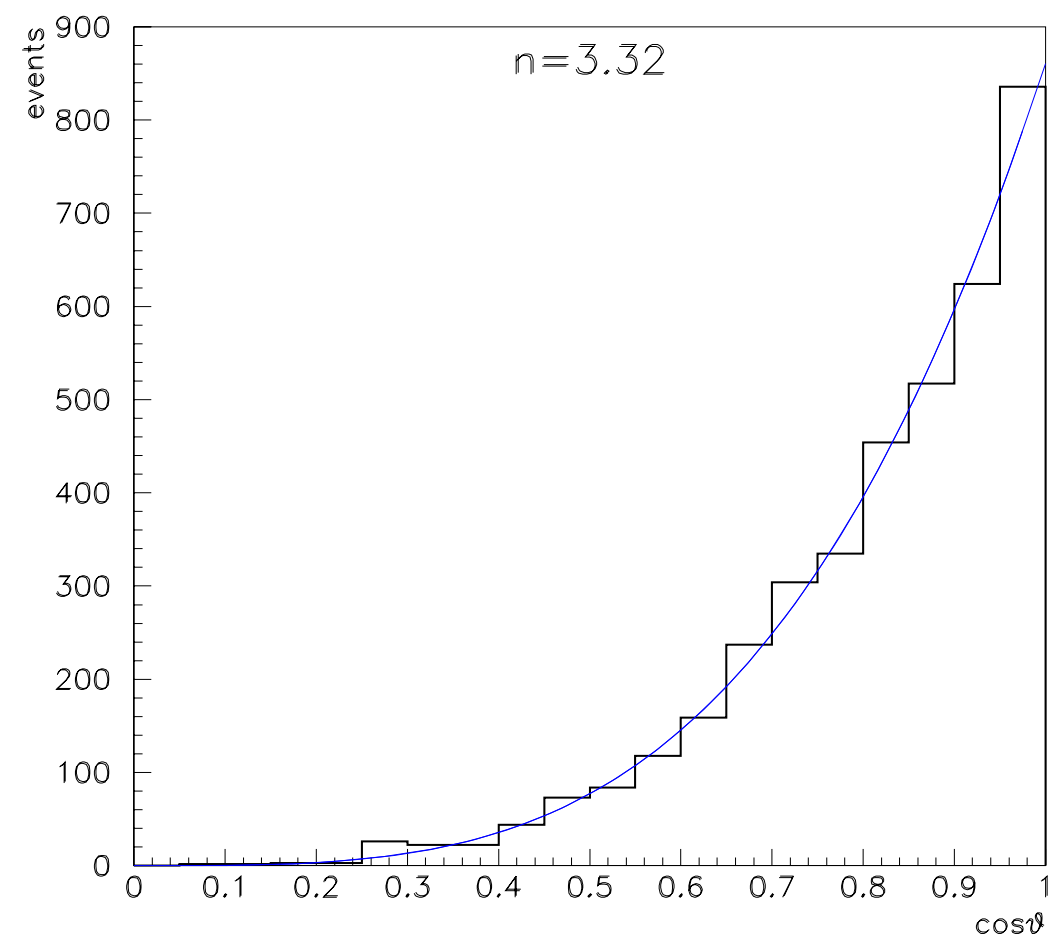

Figure 11: The zenith angle distribution of multi-muon bundles with more than two muons, compared to the simulation, which can be fitted by a power-law in $\cos ^{n} \theta$ with an exponent $n=3.32$ 

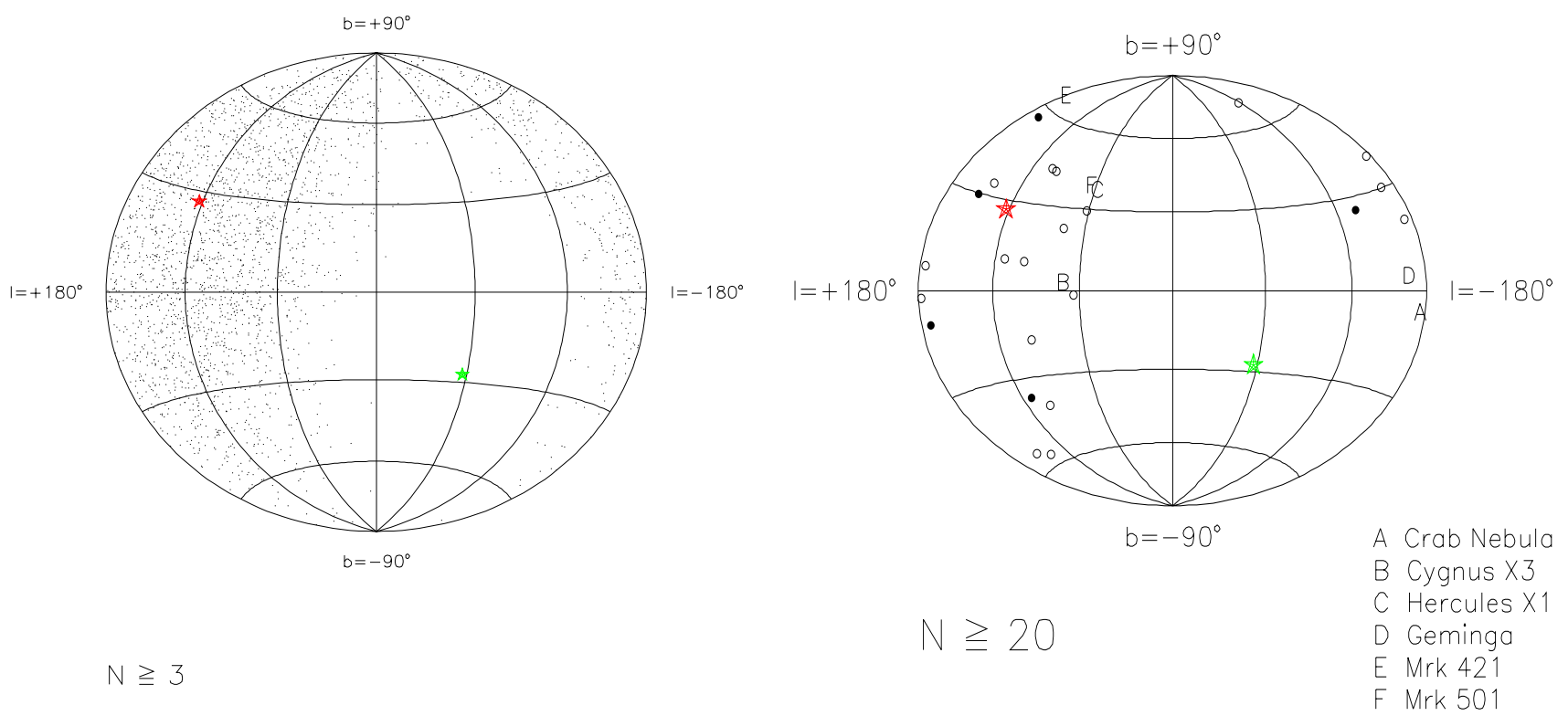

Figure 12: Apparent origins of the events on the sky in galactic coordinates for multiplicities $\geq 3$ and $\geq 20$. In the second graph the five highest multiplicity events are shown as filled circles. The asterisks indicate the positions of the Celestial North and South pole, some important known gamma ray sources visible from our detector's position are given as letters (A-D: galactic,E-F: extragalactic).

\section{Outlook}

The analysis of muon bundles observed in the ALEPH-detector provides detailed information on the density and momentum distribution of muons in the dense cores of extensive air showers underground. The interpretation of the results is further complicated for the following reasons: firstly, the chemical composition of primary cosmic rays in the energy region of interest to our analysis is essentially unknown and secondly, the interaction characteristics at energies beyond the accelerator range are not explored. Even for energies in reach of accelerators the forward production which is relevant for cosmic ray studies is poorly understood. Therefore as detailed information as possible must be collected for extensive air showers to try to disentangle the various unknowns like primary composition and their interaction characteristics. The analysis of the ALEPH cosmic ray muons was only a first step. A second step could be a larger underground array (typically $400 \mathrm{~m}^{2}$ ) with precise muon chambers complemented by a surface array to get simultaneous informations on the electromagnetic, hadronic and muonic components of extensive air showers and to study further with much larger statistics the properties of the outstanding highest multiplicity events. An experiment of this kind at CERN is in the proposal stage.

\section{Acknowledgements}

It is a pleasure for us to thank the ALEPH collaboration for the permission to use their LEP data for the cosmic ray studies and for numerous discussions with the experts about the ALEPH detector and the analysis programs. One of us (C.G.) would like to thank the "Deutsche 
Forschungsgemeinschaft" (DFG-GR-1796/1-1) for financial support.

\section{References}

[1] H.O. Klages for the KASCADE Collaboration, Proc. 25th ICRC, Durban (1997), Vol.8, 297

[2] A. Borione, C.E. Couvault, F.W. Cronin et al. NIM A 346 (1994) 329

F.W. Cronin, B.E. Fick, K.G. Gibbs et al. Phys. Rev. D45 (1992) 4385.

[3] M. Aglietta et al., for EASTOP Collaboration,Proc. 24th ICRC, Rome (1995), Vol.2, 664.

[4] MACRO Collaboration, M. Ambrosio et al., Phys. Rev. D 52,(1995) 3793.

[5] Fréjus Collaboration, Ch. Berger et al., Phys. Rev. D 40, 2163 (1989); Z. Phys. C - Particles and Fields 48, 221 (1990).

[6] Fréjus Collaboration, W. Rhode et al., Astropart. Phys. 4, 217 (1996).

[7] W.W.M. Allison et al. NIM A 376(1996) 36-48. W.W.M. Allison et al. NIM A 381(1996) 385-397. S.M. Kasahara et al. Phys. Rev. D55(1997) 5282.

[8] D. Heck et al., Report FZKA 6019 (1998), Forschungszentrum Karlsruhe J. Knapp, D. Heck, G. Schatz, Report FZKA 5828 (1996), Forschungszentrum Karlsruhe

[9] J. Hörandel for the KASCADE Collaboration, Proc. of the 16th European Cosmic Ray Symposium, July 20-24, 1998, Dept. Fisica, Universidad de Alcala, Spain (ed: J. Medina) page 579

[10] CASA-MIA: M. A. K. Glasmacher et al., Proc. 26th ICRC, Salt Lake City (1999), Vol 3, 129 and 199.

CASA-BLANCA: L. F. Fortson et al., Proc. 26th ICRC, Salt Lake City (1999), Vol 3, 125. CASA-MIA-DICE: S. P. Swordy et al., Proc. 26th ICRC, Salt Lake City (1999), Vol 3, 144.

HEGRA: A. Rohring et al, Proc. 26th ICRC, Salt Lake City (1999), Vol 3, 152.

KASCADE: K.H. Kampert et al. Proc. 26th ICRC, Salt Lake City (1999), Vol 3, 159.

J. R. Horlander et al. Proc. 26th ICRC, Salt Lake City (1999), Vol 1, 337.

J. H. Weber et al. Proc. 26th ICRC, Salt Lake City (1999), Vol 1, 341.

EAS-TOP: M. Aglietta et al.,Proc. 26th ICRC, Salt Lake City (1999), Vol 1, 230.

[11] Useful reviews include: K. Rajagopal, in Quark-Gluon Plasma 2, edited by R. Hwa, World Scientific, Singapore, (1995), p. 484; J-P. Blaizot and A. Krzywicki, Acta. Phys. Polon. B 27, 1687 (1996); K. Ragjagopal, hep-ph/9703258, talk at the International Workshop on QCD Phase Transitions, January 1997, Hirschegg, Austria; J.D. Bjorken, in Proceedings of the 1997 Zakopane School, hep-ph/9712434.

[12] J. J. Lord and J. Iwai, Paper 515, presented at the International Conference on High Energy Physics, Dallas (1992); H. Wilczynski et al., Proc. 24th ICRC, Rome (1995), Vol.1, 1. L. Pasquali, M. H. Reno, I. Sarcevic, Astroparticle Physics 9 (1998), 193.

[13] O.C. Allkofer, K. Eggert, P. Erhard et al. "The UAl detector as a possible cosmic ray device”, Proc. 17th ICRC, Paris (1981), Vol.10, 401.

[14] H. Wachsmuth, "Proposal to search for Cosmic Ray coincidences in the LEP detectors" draft, 16.Dec.1993 (CosmoLEP-note 94.000)

A. Ball, G. Bonvicini,C. Grupen, et al. CERN/LEPC 94-10, LEPC/M 109 (1994).

[15] P. Le Coultre, L3C collaboration, Proc. 25th ICRC, Durban (1997) Vol. 7, 305.

"L3+Cosmics Experiment" in "Experiments at CERN in 1998" (Grey book), p.369.

[16] D. Decamp et al., ALEPH Collaboration, NIM A 294 (1990) 121

[17] J. Ranft Cosmic Rays Particle Production hep-ph/9711334, Nucl. Phys. Proc. Suppl. 71(1999) 228. 